\title{
Necessary conditions for breathers on continuous media to approximate breathers on discrete lattices
}

\author{
WARREN R. SMITH ${ }^{1}$ and JONATHAN A. D. WATTIS ${ }^{2}$ \\ 1 School of Mathematics, The University of Birmingham, Edgbaston, Birmingham, B15 2TT, UK \\ 2 School of Mathematical Sciences, University of Nottingham, University Park, Nottingham NG7 \\ $2 R D, U K$
}

(Received 5 July 2017)

\begin{abstract}
We start by considering the sine-Gordon partial differential equation (PDE) with an arbitrary perturbation. Using the method of Kuzmak-Luke, we investigate those conditions the perturbation must satisfy in order for a breather solution to be a valid leading-order asymptotic approximation to the perturbed problem. We analyse the cases of both stationary and moving breathers. As examples, we consider perturbing terms which include typical linear damping, periodic sinusoidal driving, and dispersion caused by higher order spatial derivatives. The motivation for this study is that the mathematical modelling of physical systems, often leads to the discrete sine-Gordon system of ODEs which are then approximated in the long wavelength limit by the continuous sine-Gordon PDE. Such limits typically produce fourth-order spatial derivatives as higher order correction terms. The new results show that the stationary breather solution is a consistent solution of both the quasi-continuum SG equation and the forced/damped SG system. However, the moving breather is only a consistent solution of the quasi-continuum SG equation and not the damped SG system.
\end{abstract}

\section{Introduction}

The approximation of discrete equations by continuum versions has a long history. An important question in the use of continuum approximations, is whether the exact solution of an approximate equation is a good approximation to the solution of the original discrete problem. For example, the approximating PDE may have an exact travelling wave or breather solution; but this does not necessarily mean that the original discrete system has a travelling wave or breather. It is often assumed that while waves move through continuous systems, they do not persist in spatially discrete systems due to a PeierlsNabarro barrier.

Common applications of the discrete Klein-Gordon system include mathematical models of Josephson junctions, for example, in the work of Golubov et al [15] and Malomed [21], and models of DNA [11, 23, 28], as reviewed by Yakushevich [37]. Both Englander et al. [11] and Salerno [28] approximated the discrete sine-Gordon equation (DSG)

$$
\frac{\mathrm{d}^{2} u_{n}}{\mathrm{~d} t^{2}}=u_{n+1}-2 u_{n}+u_{n-1}-\Gamma^{2} \sin u_{n},
$$


by its continuous counterpart, which is $\phi_{t t}=\phi_{x x}-\sin \phi$, the sine-Gordon PDE (SG). This approximation process involves replacing the discrete variable $n$ by a continuous variable, $x$, and the second difference $\phi_{n+1}-2 \phi_{n}+\phi_{n-1}$ by a second derivative term, $\phi_{x x}$. By rescaling $x$ and $t$, all parameters can be removed from the sine-Gordon equation. This PDE is integrable, and has travelling wave solutions, as well as stationary and moving breather solutions. However, (1.1) is not integrable for any value of $\Gamma$. Whilst there is an integrable version of the discrete sine-Gordon equation, it arises from the 'light-cone' formulation of $\Phi_{X T}=\sin \Phi$, giving $\frac{\mathrm{d}}{\mathrm{d} t}\left(\Phi_{n+1}-\Phi_{n}\right)=\Gamma \sin \frac{1}{2}\left(\Phi_{n+1}+\Phi_{n}\right)$, as derived by Orfandis [22] and others.

The effects of discreteness in systems such as (1.1) have been studied by a number of authors, notably those interested in the dynamical systems theory perspective. Whilst it is possible for motion to occur in discrete systems, see, for example, the proof of the existence of travelling waves in the discrete and nonintegrable FPU system [13], this behaviour is far from commonplace. Peyrard and Kruskal studied the motion of a kink, and its slowing due to the discreteness of the DSG lattice [24]. More recently, Boesch and Peyrard [3] have investigated the effects of discreteness on breathers, and the more general localisation of energy has been investigated by Dauxois and Peyrard [7] and Flach and Willis [12]. Thus the DSG equation (1.1) has qualitatively different properties to that of the SG PDE.

The use of quasi-continuum techniques to study the dynamics of nonlinear lattices was initiated by Collins [5] and Collins and Rice [6], who considered the Fermi-Pasta-Ulam system, which models a chain of atoms connected by nonlinear interaction potential. This system is governed by the equation of motion $\mathrm{d}^{2} \phi_{n} / \mathrm{d} t^{2}=V^{\prime}\left(\phi_{n+1}\right)-2 V^{\prime}\left(\phi_{n}\right)+V^{\prime}\left(\phi_{n-1}\right)$. Small amplitude waves which vary slowly with $n$ can, subject to certain conditions on $V(\phi)$, be approximated by partial differential equations (PDEs). The simplest such equation is the fourth-order Boussinesq equation $\phi_{t t}=\phi_{x x}+\left(\phi^{p}\right)_{x x}+\phi_{x x x x}$, where continuous $x$ replaces the discrete $n$. However, the solitary wave solutions of this become very narrow at larger speeds, which is unphysical for a lattice approximation. The improved equations suggested by Collins [5], Collins and Rice [6], Rosenau [25, 26], have the form $\phi_{t t}=\phi_{x x}+\left(\phi^{p}\right)_{x x}+\phi_{x x t t}$.

In [35] a number of continuum versions of the discrete sine-Gordon equation (1.1) are derived, these include higher order terms from the standard Taylor series expansion of the second difference term which, when rescaled, yields the standard continuum limit

$$
u_{t t}=u_{x x}-\sin u+\varepsilon u_{x x x x}+\mathcal{O}\left(\varepsilon^{2}\right)
$$

where $\varepsilon \ll 1$. However, there are also expansions which rely on 'improved' approximating techniques PDEs, [25, 26, 32, 33] leading to

$$
\begin{aligned}
& u_{t t}=u_{x x}-\sin u+\varepsilon\left(u_{x x t t}+u_{x x} \cos u-u_{x}^{2} \sin u\right)+\mathcal{O}\left(\varepsilon^{2}\right), \\
& u_{t t}=u_{x x}-\sin u+\varepsilon\left(3 u_{x x x x}+2 u_{x x t t}+2 u_{x x} \cos u-2 u_{x}^{2} \sin u\right)+\mathcal{O}\left(\varepsilon^{3}\right) .
\end{aligned}
$$

Thus our aim is to investigate the properties of PDE approximations such as (1.2) more closely, to see if they reflect the properties of the original system (1.1) better than the simple SG PDE.

In $[33,34]$ a combination of the standard continuum limit and variational methods were used to derive approximations of breather modes in the perturbed sine-Gordon 
equation. These perturbations led to changes in the frequency-amplitude and widthamplitude relationships for breathers. In [35] a classic small amplitude multiple-scales expansion was performed on the continuum formulation, the standard continuum limit equation and on the Padé approximations above. At leading order all equations have the same solution, but at higher-order, there are subtle differences between the continuum formulation and the continuum approximations. However, we would expect these subtle differences to become more significant were larger amplitude solutions to be considered.

The complementary problem of approximating a system which is continuous in both space and time by one with discrete space and continuous time has also received attention. The approximation of continuous systems by discrete counterparts has been investigated by Kevrekedis [17] for a variety of systems, by considering carefully how to discretise each nonlinearity. The outcome of this is that the Peierls-Nabarrow potential can be removed, by ensuring that a quantity akin to momentum is conserved. Kevrekedis et al. [18] have noted that if one approximates a continuous Klein-Gordon equation by a the expected discretisation, then the discrete momentum is not conserved. Hence they propose adding a perturbation term to the discrete system which ensures conservation of momentum. However, this is at the expense of the energy no longer being constant. Dmitriev et al. [9] have pointed out that for the Klein-Gordon system, the additional terms required to conserve momentum, destroy the conservation of energy cause and permit accelerating waves to occur. In addition, Cisneros-Ake [4] has used variational approximations to model the motion of dislocations in lattices using both spatially discrete and continuous descriptions. He finds the shape of the kink solutions to be similar, but significant differences in the shape of the dispersion relation.

Perturbation theory based on the inverse scattering transform has been previously applied to the moving breather (see, for example, [16]). This technique differs from the applied mathematics literature on oscillatory waves. Firstly, approximate breathers are sought in a restricted family of solutions which lack the long length scale. Secondly, local wave number and frequency are not introduced and conservation of waves does not form part of the analysis [36]. The results of this inverse scattering analysis will be compared with our asymptotic analysis of the moving breather which is consistent with the applied mathematics literature on oscillatory waves.

Herein, we use the method of Kuzmak-Luke [19, 20] to analyse the perturbed sineGordon equation for large-amplitude stationary and moving breathers. The stationary breather is a nonlinear oscillator with spatial dependence, the only previous analysis of this type is for the damped oscillations of an incompressible viscous drop [31]. The moving breather corresponds to a wave with two short scales in which the local frequency and local wave number are correlated. Our analysis of this particular wave is the first of its type; nevertheless, it has several features in common with the single-phased Klein-Gordon wave studied in [30].

Breathers have been shown not to persist for the perturbed sine-Gordon equation: they very slowly radiate their energy due to exponentially small terms (see $[8,14,29])$. Our approximate analysis of stationary and moving breathers takes place on a time scale of order $1 / \epsilon$ which is much shorter than the extremely slow decay due to radiation, where $\epsilon$ is the small parameter. Exponentially small terms are irrelevant on an order $1 / \epsilon$ time scale. Furthermore, numerical simulations have shown that, over long time scales, breathers of 
the perturbed sine-Gordon equation may also blow up or split into a kink-antikink pair (see $[1,2]$ and references therein). The following asymptotic analysis will only be valid when breathers are stable solutions.

In the next section, the asymptotic analysis of the stationary breather is performed for an arbitrary perturbation. The perturbations to the continuous sine-Gordon system in the continuum versions of the discrete sine-Gordon equation are shown to be nondissipative and the effects of small-amplitude damping in a discrete lattice such as DNA are investigated. Section 3 describes the more challenging asymptotic analysis of the moving breather. The perturbations in the continuum versions of the discrete sine-Gordon equation are also shown to be non-dissipative in this case. However, the moving breather is found to be incompatible with damping in contrast to the stationary breather. Finally, in Section 4 we discuss the results, and compare them with the results of others' work.

\section{Stationary breather}

We consider perturbed sine-Gordon equations of the form

$$
\frac{\partial^{2} u}{\partial t^{2}}-\frac{\partial^{2} u}{\partial x^{2}}+\sin (u)=\epsilon F\left(x, t, u, \frac{\partial u}{\partial x}, \frac{\partial u}{\partial t}, \ldots\right)
$$

with the boundary conditions that

$$
u \text { decays to zero exponentially as } x \rightarrow \pm \infty,
$$

in which $0<\epsilon \ll 1$ and $F$ is an arbitrary perturbation function. Furthermore we assume that the boundary value problem (2.1)-(2.2) with $\epsilon=0$ has time periodic solutions.

\subsection{The leading-order solution}

For $\epsilon \ll 1$, the frequency of the stationary breather is approximately constant when $t=\mathcal{O}(1)$. However, over a time scale of $t=\mathcal{O}(1 / \epsilon)$, the variation of the frequency is an order one effect. In order to capture this effect over the $1 / \epsilon$ time scale, we adopt a fast time scale $t^{+}$and a slow time scale $\widetilde{t}$ with

$$
\frac{\mathrm{d} t^{+}}{\mathrm{d} t}=\omega(\widetilde{t}), \quad \widetilde{t}=\epsilon t
$$

where $\omega(\widetilde{t})$ is the frequency of oscillation. The main idea behind Kuzmak's approach is that the leading order solution, $u_{0}$ is periodic in $t^{+}$, with period precisely $2 \pi$, so that if the perturbation $F$ causes the frequency of oscillation to change this is accounted for by changing the frequency $\omega(\widetilde{t})$ and not by altering $u_{0}$. We introduce expansions of the form

$$
u \sim u_{0}\left(t^{+}, x, \widetilde{t}\right)+\epsilon u_{1}\left(t^{+}, x, \widetilde{t}\right), \quad F \sim F_{0}\left(t^{+}, x, \widetilde{t}\right)+\epsilon F_{1}\left(t^{+}, x, \widetilde{t}\right),
$$

as $\epsilon \rightarrow 0$. The leading-order problem is given by

$$
\omega^{2} \frac{\partial^{2} u_{0}}{\partial t^{+2}}-\frac{\partial^{2} u_{0}}{\partial x^{2}}+\sin \left(u_{0}\right)=0,
$$

with the boundary condition

$$
u_{0} \text { decays to zero exponentially as } x \rightarrow \pm \infty \text {, }
$$


and the periodicity condition

$$
u_{0}\left(t^{+}+\Psi, x, \widetilde{t}\right)=u_{0}\left(t^{+}+\Psi-2 \pi, x, \widetilde{t}\right),
$$

where $\Psi(\widetilde{t})$ is the phase shift. This problem is readily integrated to yield the stationary breather solution

$$
u_{0}=4 \arctan \left(\frac{\left(1-\omega^{2}\right)^{1 / 2} \cos \left(t^{+}+\Psi\right)}{\omega \cosh \left(\left(1-\omega^{2}\right)^{1 / 2}(x+\Lambda)\right)}\right) .
$$

The slowly varying phase shift $\Psi(\widetilde{t})$ and the constant $\Lambda$ should be incorporated into (2.6), $\Lambda$ corresponding to the arbitrariness of the origin of $x$ in (2.3)-(2.5). We note that $u_{0}$ is even about $t^{+}+\Psi=n \pi$ and about $x+\Lambda=0$, where $n$ is an integer. The former parity condition corresponds to

$$
u_{0}\left(t^{+}+\Psi, x+\Lambda, \widetilde{t}\right)=u_{0}\left(2 \pi-\left(t^{+}+\Psi\right), x+\Lambda, \widetilde{t}\right)
$$

in combination with the periodicity condition (2.5), whereas the latter parity condition corresponds to

$$
u_{0}\left(t^{+}+\Psi, x+\Lambda, \widetilde{t}\right)=u_{0}\left(t^{+}+\Psi,-(x+\Lambda), \widetilde{t}\right) .
$$

The amplitude envelope, which in this problem is only governed by the frequency of oscillation, is of interest in the subsequent analysis.

\subsection{The first correction}

At next order we have

$$
\omega^{2} \frac{\partial^{2} u_{1}}{\partial t^{+2}}-\frac{\partial^{2} u_{1}}{\partial x^{2}}+\cos \left(u_{0}\right) u_{1}=F_{0}-2 \omega \frac{\partial^{2} u_{0}}{\partial \widetilde{t} \partial t^{+}}-\frac{\mathrm{d} \omega}{\mathrm{d} \widetilde{t}} \frac{\partial u_{0}}{\partial t^{+}}
$$

with the boundary condition

$$
u_{1} \text { decays to zero exponentially as } x \rightarrow \pm \infty,
$$

and the periodicity condition

$$
u_{1}\left(t^{+}+\Psi, x, \widetilde{t}\right)=u_{1}\left(t^{+}+\Psi-2 \pi, x, \widetilde{t}\right),
$$

following the equations (2.4)-(2.5). The Fredholm alternative is now applied to this linear problem (2.7)-(2.9). We define

$$
\langle\cdot\rangle=\int_{t^{+}=-\Psi}^{2 \pi-\Psi} \int_{x=-\infty}^{\infty} \cdot \mathrm{d} x \mathrm{~d} t^{+} .
$$

A function $v$ in the null space of the adjoint problem satisfies

$$
\omega^{2} \frac{\partial^{2} v}{\partial t^{+2}}-\frac{\partial^{2} v}{\partial x^{2}}+\cos \left(u_{0}\right) v=0
$$

subject to the boundary condition

$$
v \rightarrow 0 \text { as } x \rightarrow \pm \infty,
$$

and the periodicity condition

$$
v\left(t^{+}+\Psi, x, \widetilde{t}\right)=v\left(t^{+}+\Psi-2 \pi, x, \widetilde{t}\right) .
$$


Our linear problem for the first correction (2.7)-(2.9) can only have a solution if

$$
\frac{\mathrm{d} \omega}{\mathrm{d} \widetilde{t}}\left\langle v \frac{\partial u_{0}}{\partial t^{+}}\right\rangle+2 \omega\left\langle v \frac{\partial^{2} u_{0}}{\partial \widetilde{t} \partial t^{+}}\right\rangle=\left\langle v F_{0}\right\rangle
$$

for any $v$ in the null space.

Two linearly independent solutions of the adjoint problem (2.10)-(2.12) have been determined

$$
v_{1}=\frac{\partial u_{0}}{\partial t^{+}}, \quad v_{2}=\frac{\partial u_{0}}{\partial x}
$$

where the first solution is odd about $t^{+}+\Psi=n \pi$ and the second even. Since $u_{0}$ is even about $x+\Lambda=0$, so is $\partial u_{0} / \partial t^{+}$, and $\partial u_{0} / \partial x$ is odd about $x+\Lambda=0$. The first solution corresponds to an amplitude modulation equation and the second a solvability condition associated with the stationary breather. The equation for the modulation of the phase shift $\Psi(\widetilde{t})$ requires consideration of the problem at $\mathcal{O}\left(\epsilon^{2}\right)$.

\subsubsection{Amplitude modulation equation}

If we substitute the first solution $v_{1}$ into (2.13), we obtain the equation

$$
\frac{\mathrm{d} \omega}{\mathrm{d} \widetilde{t}}\left\langle\left(\frac{\partial u_{0}}{\partial t^{+}}\right)^{2}\right\rangle+2 \omega\left\langle\frac{\partial u_{0}}{\partial t^{+}} \frac{\partial^{2} u_{0}}{\partial \widetilde{t} \partial t^{+}}\right\rangle=\left\langle\frac{\partial u_{0}}{\partial t^{+}} F_{0}\right\rangle .
$$

We define the wave action

in order to rewrite the our modulation equation as

$$
J(\omega(\widetilde{t}))=\frac{\omega}{2}\left\langle\left(\frac{\partial u_{0}}{\partial t^{+}}\right)^{2}\right\rangle
$$

$$
\frac{\mathrm{d} J}{\mathrm{~d} \widetilde{t}}=\frac{1}{2}\left\langle\frac{\partial u_{0}}{\partial t^{+}} F_{0}\right\rangle
$$

We note that the wave action defined above is distinct from the action which is usually defined in the Lagrangian-Hamiltonian derivation. In order to simplify the wave action, we write

$$
J=8 \Omega \int_{\xi=-\infty}^{\infty} \operatorname{sech}^{2}(\xi) I(\xi, \omega(\widetilde{t})) \mathrm{d} \xi
$$

in which $\Omega=\left(1-\omega^{2}\right)^{1 / 2} / \omega$ and

$$
I=\int_{t^{+}=-\Psi}^{2 \pi-\Psi} \frac{\sin ^{2}\left(t^{+}+\Psi\right)}{\left(1+\Omega^{2} \operatorname{sech}^{2}(\xi) \cos ^{2}\left(t^{+}+\Psi\right)\right)^{2}} \mathrm{~d} t^{+} .
$$

Using the substitution $z=e^{i\left(t^{+}+\Psi\right)}$ and integrating around the unit circle in the complex plane, we obtain

and

$$
I=\frac{\pi}{\left(\Omega^{2} \operatorname{sech}^{2}(\xi)+1\right)^{1 / 2}}
$$

$$
J=8 \pi \Omega \int_{\xi=-\infty}^{\infty} \frac{\operatorname{sech}^{2}(\xi)}{\left(\Omega^{2} \operatorname{sech}^{2}(\xi)+1\right)^{1 / 2}} \mathrm{~d} \xi
$$


We now introduce the substitution $y=\tanh (\xi)$ to yield $J=16 \pi \arccos (\omega)$ or

$$
\omega=\cos (J / 16 \pi),
$$

provided that $J<8 \pi^{2}$. The amplitude envelope may be expressed in simplified form

$$
u_{\max }(x, \widetilde{t})=4 \arctan \left(\frac{\tan (J / 16 \pi)}{\cosh (\sin (J / 16 \pi)[x+\Lambda])}\right) .
$$

\subsubsection{Solvability condition}

If we substitute the second solution $v_{2}$ into (2.13), we have

$$
\frac{\mathrm{d} \omega}{\mathrm{d} \widetilde{t}}\left\langle\frac{\partial u_{0}}{\partial x} \frac{\partial u_{0}}{\partial t^{+}}\right\rangle+2 \omega\left\langle\frac{\partial u_{0}}{\partial x} \frac{\partial^{2} u_{0}}{\partial \widetilde{t} \partial t^{+}}\right\rangle=\left\langle\frac{\partial u_{0}}{\partial x} F_{0}\right\rangle .
$$

The first term on the left-hand side of this equation contains the product of even and odd functions integrated over one period of oscillation, this being zero. The second term on the left-hand side requires further analysis. The structure of $u_{0}$ takes the form (in view of (2.6))

$$
u_{0}=u_{0}\left(t^{+}+\Psi(\widetilde{t}), x ; \omega(\widetilde{t})\right) .
$$

This structure may be differentiated to yield

$$
\frac{\partial^{2} u_{0}}{\partial \widetilde{t} \partial t^{+}}=\frac{\partial^{2} u_{0}}{\partial \omega \partial t^{+}} \frac{\mathrm{d} \omega}{\mathrm{d} \widetilde{t}}+\frac{\partial^{2} u_{0}}{\partial t^{+2}} \frac{\mathrm{d} \Psi}{\mathrm{d} \widetilde{t}}
$$

where the first term on the right-hand side is odd in $t^{+}+\Psi$ and the second even. We substitute and exploit parity arguments again to obtain

$$
2 \omega \frac{\mathrm{d} \Psi}{\mathrm{d} \widetilde{t}}\left\langle\frac{\partial u_{0}}{\partial x} \frac{\partial^{2} u_{0}}{\partial t^{+2}}\right\rangle=\left\langle\frac{\partial u_{0}}{\partial x} F_{0}\right\rangle .
$$

This equation may be further simplified by substitution of (2.3) and integrating in $x$ to yield the solvability condition

$$
\left\langle\frac{\partial u_{0}}{\partial x} F_{0}\right\rangle=0
$$

The amplitude modulation equation (2.14) and solvability equation (2.17) are necessary conditions for the linear problem for the first correction (2.7)-(2.9) to have a solution.

\subsection{Necessary conditions}

If the continuous sine-Gordon equation is to be a valid approximation, then the amplitude envelope should remain constant. The two necessary conditions for stationary breather solutions on continuous media to represent breather solutions on discrete lattices are

$$
\left\langle\frac{\partial u_{0}}{\partial t^{+}} F_{0}\right\rangle=0, \quad\left\langle\frac{\partial u_{0}}{\partial x} F_{0}\right\rangle=0 .
$$

In the continuum version (1.2), we have

$$
F_{0}=\frac{\partial^{4} u_{0}}{\partial x^{4}}
$$


which is even about $t^{+}+\Psi=n \pi$ and $x+\Lambda=0$. The left-hand side of the first condition in (2.18) is the product of an odd and even function in $t^{+}+\Psi$ integrated over the period of oscillation, this being zero. The left-hand side of the second condition in (2.18) is the product of an odd and even function in $x+\Lambda$ integrated over the real line, which is also zero. Both necessary conditions are met and the amplitude envelope remains constant. As the solutions in [30] demonstrate, the phase shift $\Psi(\widetilde{t})$ may still vary even when the frequency and amplitude are constant.

If we consider the continuum reformulations to the discrete operator obtained using Padé approximations (1.3) and (1.4), then

$$
F_{0}=\omega^{2} \frac{\partial^{4} u_{0}}{\partial x^{2} \partial t^{+2}}+\frac{\partial^{2} u_{0}}{\partial x^{2}} \cos \left(u_{0}\right)-\left(\frac{\partial u_{0}}{\partial x}\right)^{2} \sin \left(u_{0}\right)
$$

and

$$
F_{0}=3 \frac{\partial^{4} u_{0}}{\partial x^{4}}+2 \omega^{2} \frac{\partial^{4} u_{0}}{\partial x^{2} \partial t^{+2}}+2 \frac{\partial^{2} u_{0}}{\partial x^{2}} \cos \left(u_{0}\right)-2\left(\frac{\partial u_{0}}{\partial x}\right)^{2} \sin \left(u_{0}\right)
$$

respectively. We have $F_{0}$ even about $t^{+}+\Psi=n \pi$ and $x+\Lambda=0$ in both of these cases. The conditions in (2.18) are met following the same parity argument as above. Thus, it is quite feasible for a static breather solution to be the large-time asymptotic solution in this case. This is consistent with the existing theory in which, in the anti-continuum, limit stationary breathers can be rigorously proven to exist in discrete Klein-Gordon systems.

\subsection{Numerical method}

In this subsection, an accurate numerical method for the perturbed discrete SG equation is described. We consider the system of ordinary differential equations

$$
\frac{\mathrm{d}^{2} \widehat{u}_{n}}{\mathrm{~d} t^{2}}=\widehat{u}_{n+1}-2 \widehat{u}_{n}+\widehat{u}_{n-1}-\sin \left(\widehat{u}_{n}\right)+\epsilon \widehat{F}_{n}
$$

where $-N+1 \leq n \leq N-1$ and $\widehat{F}_{n}$ is a perturbation centred at $n$. The nodes neglected in this truncation are modelled by the discrete one-way wave equations

$$
\frac{\mathrm{d} \widehat{u}_{-N}}{\mathrm{~d} t}=\widehat{u}_{-N+1}-\widehat{u}_{-N}, \quad \frac{\mathrm{d} \widehat{u}_{N}}{\mathrm{~d} t}=\widehat{u}_{N-1}-\widehat{u}_{N}
$$

The coupled system of equations (2.19)-(2.20) is solved using the NAG routine D02EJF.

The discrete stationary breather is investigated using the initial conditions

$$
\widehat{u}_{n}(0)=4 \arctan \left(\frac{\left(1-\omega^{2}\right)^{1 / 2}}{\omega \cosh \left(\left(1-\omega^{2}\right)^{1 / 2} n\right)}\right), \quad \frac{\mathrm{d} \widehat{u}_{n}}{\mathrm{~d} t}(0)=0,
$$

with the corresponding initial conditions being taken for the continuum version, in which $\omega(0)=\cos (\pi / 16)$. In order to validate the numerical method, our first problem concerns the unperturbed discrete problem and the asymptotic envelope of (1.2). As both of these problems are Hamiltonian, constant amplitudes should be anticipated. The results of the numerical simulation and the asymptotic envelope are shown in Figure 1, the agreement being excellent. 

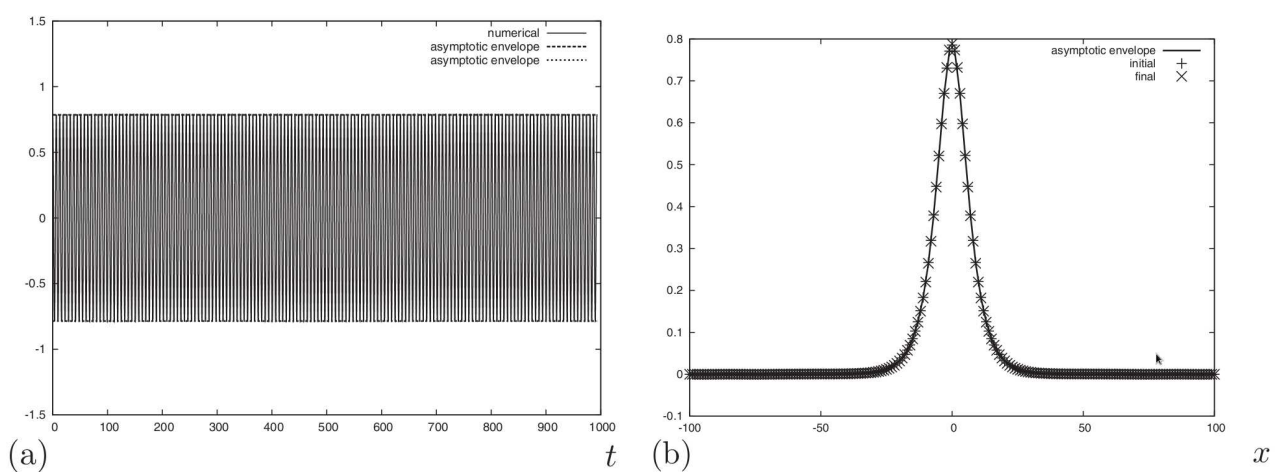

FiguRE 1. Stationary breather integrated over an integer number of periods with $\epsilon=0.1$ : (a) $\widehat{u}_{0}(t)$ and its asymptotic envelope $u_{\max }(0, t)$ for $0 \leq t \leq t_{\text {end }}$ and (b) $\widehat{u}_{n}(0), \widehat{u}_{n}\left(t_{\text {end }}\right)$ and $u_{\text {max }}\left(x, t_{\text {end }}\right)$.

\subsection{DNA simulation}

A stationary breather on the discrete lattice of DNA may be simulated by perturbations of the form $F=u_{x x x x}-\lambda u_{t}+\alpha \sin (\bar{\omega} t)$ in $(2.1)$ and $\widehat{F}_{n}=-\lambda \mathrm{d} \widehat{u}_{n} / \mathrm{d} t+\alpha \sin (\bar{\omega} t)$ in (2.19), where $\lambda>0$ and $\alpha>0$. We have

$$
F_{0}=\frac{\partial^{4} u_{0}}{\partial x^{4}}-\lambda \omega \frac{\partial u_{0}}{\partial t^{+}}+\alpha \sin (\bar{\omega} t)
$$

The first term reproduces the effect of discreteness, the second models damping and the third represents a small amplitude background forcing in DNA [37]. The slow time scale, $\widetilde{t}$, is based on the first term in the perturbation, whereas the effect of damping or forcing is small on this "discreteness" time scale. The amplitude modulation equation (2.14) becomes

$$
\frac{\mathrm{d} J}{\mathrm{~d} \widetilde{t}}+\lambda J=\frac{\alpha}{2}\left\langle\frac{\partial u_{0}}{\partial t^{+}} \sin (\bar{\omega} t)\right\rangle
$$

in which

$$
t^{+}=\frac{1}{\epsilon} \int_{s=0}^{\epsilon t} \omega(s) \mathrm{d} s
$$

The solvability condition (2.17) is satisfied by considering parity. Equation (2.21) is not amenable to analytical solution. Henceforth, we consider the damped breather $(\alpha=0)$, then $(2.21)$ is readily integrated to yield

$$
J(\omega(\widetilde{t}))=J(\omega(0)) e^{-\lambda \widetilde{t}} .
$$

On the long time scale, we have an exponential decay. Using (2.15), the modulated frequency is given by

$$
\omega(\widetilde{t})=\cos \left(\frac{J(\omega(0))}{16 \pi} e^{-\lambda \widetilde{t}}\right) .
$$

In order to validate the results, we consider $\epsilon=0.1$ and $\lambda=0.01$ and the initial condition $J(\omega(0))=\pi^{2}$ or $\omega(0)=\cos (\pi / 16)$. The slow modulation of amplitude in the discrete SG equation and its continuum version are compared in Figure 2, the agreement being excellent. 


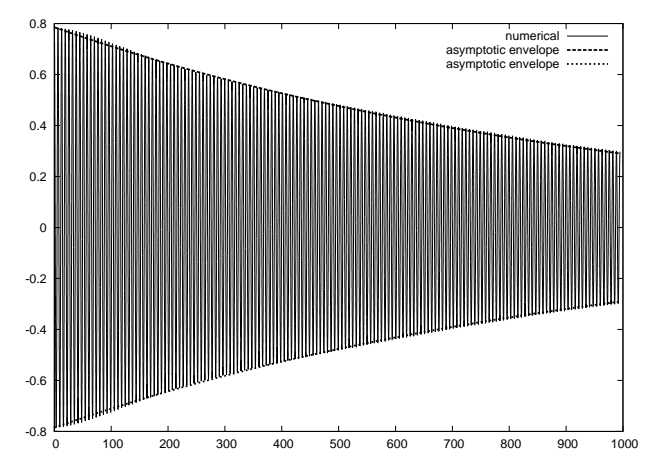

(a)

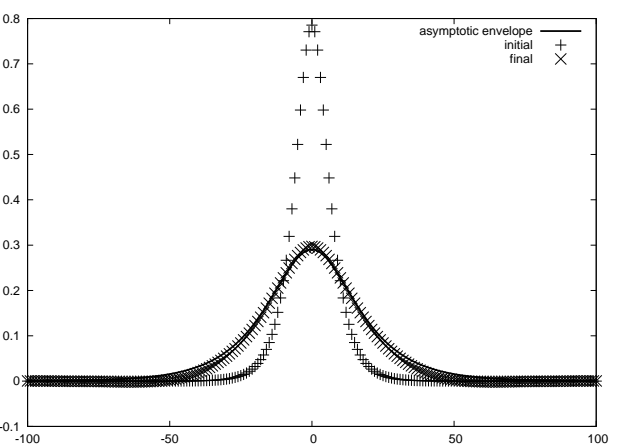

(b)

$\lambda=0.01$ : (a) $\widehat{u}_{0}(t)$ and its asymptotic envelope $u_{\max }(0, t)$ for $0 \leq t \leq t_{\text {end }}$ and (b) $\widehat{u}_{n}(0)$, $\widehat{u}_{n}\left(t_{\text {end }}\right)$ and $u_{\max }\left(x, t_{\text {end }}\right)$.

\section{Moving breathers}

We again consider the perturbed sine-Gordon equations (2.1) with the boundary conditions (2.2). We assume that the initial conditions with $\epsilon=0$ are consistent with moving breather solutions which are exactly periodic.

\subsection{The leading-order solution}

For $\epsilon \ll 1$, the local frequency and the local wave number of the moving breather are approximately constant when $t=\mathcal{O}(1)$ and $x=\mathcal{O}(1)$. However, over a time scale of $t=\mathcal{O}(1 / \epsilon)$ or a length scale of $x=\mathcal{O}(1 / \epsilon)$, the variation of the local frequency and the local wave number is an order one effect. In order to capture this effect over the $1 / \epsilon$ time and length scales, we adopt two fast scales $\theta$ and $\phi$ defined by

$$
\theta_{x}=\frac{\omega k}{\mu}, \quad \theta_{t}=-\frac{\omega^{2}}{\mu}, \quad \phi_{x}=\frac{\omega}{\mu} \text { and } \phi_{t}=-\frac{k}{\mu},
$$

where $\mu=\sqrt{\omega^{2}-k^{2}}$, the slow time scale $\widetilde{t}=\epsilon t$, the slow length scale $\widetilde{x}=\epsilon x, k=k(\widetilde{x}, \widetilde{t})$ and $\omega=\omega(\widetilde{x}, \widetilde{t})$. The level surfaces of $\theta$ and $\phi$ can be recognized as waves with two distinct slowly varying wave speeds. The moving breather will be periodic in $\theta$ with local wave number $\omega k / \mu$, local frequency $\omega^{2} / \mu$ and local phase velocity $\omega / k$. The short scale $\phi$ is required to model the second slowly varying wave speed $k / \omega$. The moving breather is not periodic in $\phi$; however, the solution is exponentially small outside a short interval. These definitions are a generalization of the definition of the local wave number and local frequency for a strongly nonlinear wave train (see, for example, [36]), this formulation being based on the Lorentz invariance of the unperturbed problem. If $k=0$, then we recover the fast scales for the stationary breather except that $\theta_{t}=-\omega$. The definitions of $\theta$ and $\phi$ given by (3.1) are consistent only if

$$
\frac{\partial}{\partial t}\left(\theta_{x}\right)=\frac{\partial}{\partial x}\left(\theta_{t}\right), \quad \frac{\partial}{\partial t}\left(\phi_{x}\right)=\frac{\partial}{\partial x}\left(\phi_{t}\right)
$$


that is,

$$
\frac{\partial}{\partial \widetilde{t}}\left(\frac{\omega k}{\mu}\right)+\frac{\partial}{\partial \widetilde{x}}\left(\frac{\omega^{2}}{\mu}\right)=0, \quad \frac{\partial}{\partial \widetilde{t}}\left(\frac{\omega}{\mu}\right)+\frac{\partial}{\partial \widetilde{x}}\left(\frac{k}{\mu}\right)=0,
$$

respectively. We introduce expansions of the form

$$
u \sim u_{0}(\phi, \theta, \widetilde{x}, \widetilde{t})+\epsilon u_{1}(\phi, \theta, \widetilde{x}, \widetilde{t}), \quad F \sim F_{0}(\phi, \theta, \widetilde{x}, \widetilde{t})+\epsilon F_{1}(\phi, \theta, \widetilde{x}, \widetilde{t}),
$$

as $\epsilon \rightarrow 0$. The leading-order problem is given by

$$
\omega^{2} \frac{\partial^{2} u_{0}}{\partial \theta^{2}}-\frac{\partial^{2} u_{0}}{\partial \phi^{2}}+\sin \left(u_{0}\right)=0
$$

The boundary condition becomes

$$
u_{0} \text { decays to zero exponentially as } \phi \rightarrow \pm \infty \text {, }
$$

and the periodicity condition

$$
u_{0}(\phi+\Lambda, \theta+\Psi, \widetilde{x}, \widetilde{t})=u_{0}(\phi+\Lambda, \theta+\Psi-2 \pi, \widetilde{x}, \widetilde{t})
$$

where $\Lambda(\widetilde{x}, \widetilde{t})$ is a slowly varying parameter and $\Psi(\widetilde{x}, \widetilde{t})$ is the phase shift. The moving breather integral of this problem is given by

$$
u_{0}=4 \arctan \left(\frac{\left(1-\omega^{2}\right)^{1 / 2} \cos (\theta+\Psi)}{\omega \cosh \left(\left(1-\omega^{2}\right)^{1 / 2}(\phi+\Lambda)\right)}\right) .
$$

We note that $u_{0}$ is even about $\theta+\Psi=n \pi$ and about $\phi+\Lambda=0$, where $n$ is an integer. In (3.4), $\theta$ and $\phi$ represent time-like and space-like variables, respectively. The amplitude envelope, which in this problem is only governed by $\omega$, is of interest in the subsequent analysis.

\subsection{The first correction}

At next order we have

$$
\begin{aligned}
& \omega^{2} \frac{\partial^{2} u_{1}}{\partial \theta^{2}}-\frac{\partial^{2} u_{1}}{\partial \phi^{2}}+\cos \left(u_{0}\right) u_{1} \\
& =F_{0}+2\left(\frac{\omega^{2}}{\mu} \frac{\partial^{2} u_{0}}{\partial \widetilde{t} \partial \theta}+\frac{k}{\mu} \frac{\partial^{2} u_{0}}{\partial \widetilde{t} \partial \phi}+\frac{\omega k}{\mu} \frac{\partial^{2} u_{0}}{\partial \widetilde{x} \partial \theta}+\frac{\omega}{\mu} \frac{\partial^{2} u_{0}}{\partial \widetilde{x} \partial \phi}\right) \\
& +\frac{\partial}{\partial \widetilde{t}}\left(\frac{\omega^{2}}{\mu}\right) \frac{\partial u_{0}}{\partial \theta}+\frac{\partial}{\partial \widetilde{t}}\left(\frac{k}{\mu}\right) \frac{\partial u_{0}}{\partial \phi}+\frac{\partial}{\partial \widetilde{x}}\left(\frac{\omega k}{\mu}\right) \frac{\partial u_{0}}{\partial \theta}+\frac{\partial}{\partial \widetilde{x}}\left(\frac{\omega}{\mu}\right) \frac{\partial u_{0}}{\partial \phi},
\end{aligned}
$$

with the boundary condition

$$
u_{1} \text { decays to zero exponentially as } \phi \rightarrow \pm \infty,
$$

and the periodicity condition

$$
u_{1}(\phi+\Lambda, \theta+\Psi, \widetilde{x}, \widetilde{t})=u_{1}(\phi+\Lambda, \theta+\Psi-2 \pi, \widetilde{x}, \widetilde{t}) .
$$

As for the stationary breather, the Fredholm alternative is applied to this linear problem (3.5)-(3.7). We define

$$
\langle\cdot\rangle=\int_{\theta=-\Psi}^{2 \pi-\Psi} \int_{\phi=-\infty}^{\infty} \cdot \mathrm{d} \phi \mathrm{d} \theta
$$


A function $v$ in the null space of the adjoint problem satisfies

$$
\omega^{2} \frac{\partial^{2} v}{\partial \theta^{2}}-\frac{\partial^{2} v}{\partial \phi^{2}}+\cos \left(u_{0}\right) v=0
$$

subject to the boundary condition

$$
v \rightarrow 0 \text { as } \phi \rightarrow \pm \infty
$$

and the periodicity condition

$$
v(\phi+\Lambda, \theta+\Psi, \widetilde{x}, \widetilde{t})=v(\phi+\Lambda, \theta+\Psi-2 \pi, \widetilde{x}, \widetilde{t}) .
$$

Our linear problem for the first correction (3.5)-(3.7) can only have a solution if

$$
\begin{aligned}
& \left\langle v\left[\frac{\partial}{\partial \widetilde{t}}\left(\frac{\omega^{2}}{\mu}\right) \frac{\partial u_{0}}{\partial \theta}+\frac{\partial}{\partial \widetilde{t}}\left(\frac{k}{\mu}\right) \frac{\partial u_{0}}{\partial \phi}+\frac{\partial}{\partial \widetilde{x}}\left(\frac{\omega k}{\mu}\right) \frac{\partial u_{0}}{\partial \theta}+\frac{\partial}{\partial \widetilde{x}}\left(\frac{\omega}{\mu}\right) \frac{\partial u_{0}}{\partial \phi}\right]\right\rangle \\
& +2\left\langle v\left[\frac{\omega^{2}}{\mu} \frac{\partial^{2} u_{0}}{\partial \widetilde{t} \partial \theta}+\frac{k}{\mu} \frac{\partial^{2} u_{0}}{\partial \widetilde{t} \partial \phi}+\frac{\omega k}{\mu} \frac{\partial^{2} u_{0}}{\partial \widetilde{x} \partial \theta}+\frac{\omega}{\mu} \frac{\partial^{2} u_{0}}{\partial \widetilde{x} \partial \phi}\right]\right\rangle=-\left\langle v F_{0}\right\rangle,
\end{aligned}
$$

for any $v$ in the null space.

Two linearly independent solutions of the adjoint problem (3.8)-(3.10) have been determined

$$
v_{1}=\frac{\partial u_{0}}{\partial \theta}, \quad v_{2}=\frac{\partial u_{0}}{\partial \phi}
$$

where the first solution is odd about $\theta+\Psi=n \pi$ and the second even. Since $u_{0}$ is even about $\phi+\Lambda=0$, so is $\partial u_{0} / \partial \theta$, and $\partial u_{0} / \partial \phi$ is odd about $\phi+\Lambda=0$. The first solution corresponds to an amplitude modulation equation and the second a solvability condition associated with the moving breather.

\subsubsection{Amplitude modulation equation}

In view of (3.4), the structure of $u_{0}$ takes the form

$$
u_{0}=u_{0}(\phi+\Lambda(\widetilde{x}, \widetilde{t}), \theta+\Psi(\widetilde{x}, \widetilde{t}) ; \omega(\widetilde{x}, \widetilde{t})) .
$$

This structure may be differentiated to yield

$$
\begin{aligned}
& \frac{\partial^{2} u_{0}}{\partial \widetilde{t} \partial \theta}=\frac{\partial^{2} u_{0}}{\partial \phi \partial \theta} \frac{\partial \Lambda}{\partial \widetilde{t}}+\frac{\partial^{2} u_{0}}{\partial \theta^{2}} \frac{\partial \Psi}{\partial \widetilde{t}}+\frac{\partial^{2} u_{0}}{\partial \omega \partial \theta} \frac{\partial \omega}{\partial \widetilde{t}} \\
& \frac{\partial^{2} u_{0}}{\partial \widetilde{t} \partial \phi}=\frac{\partial^{2} u_{0}}{\partial \phi^{2}} \frac{\partial \Lambda}{\partial \widetilde{t}}+\frac{\partial^{2} u_{0}}{\partial \phi \partial \theta} \frac{\partial \Psi}{\partial \widetilde{t}}+\frac{\partial^{2} u_{0}}{\partial \omega \partial \phi} \frac{\partial \omega}{\partial \widetilde{t}}
\end{aligned}
$$

The first and third terms on the right-hand side of (3.12) and the second term on the right-hand side of (3.13) are odd about $\theta+\Psi=n \pi$; the remaining terms on the righthand sides of (3.12)-(3.13) are even. The first term on the right-hand side of (3.12) and the second and the third terms on the right-hand side of (3.13) are odd about $\phi+\Lambda=0$; the remaining terms on the right-hand sides of (3.12)-(3.13) are even. A similar result may be obtained by differentiating with respect to $\widetilde{x}$.

If we substitute the first solution $v_{1}$ into (3.11) and exploit the parity of the terms, we 


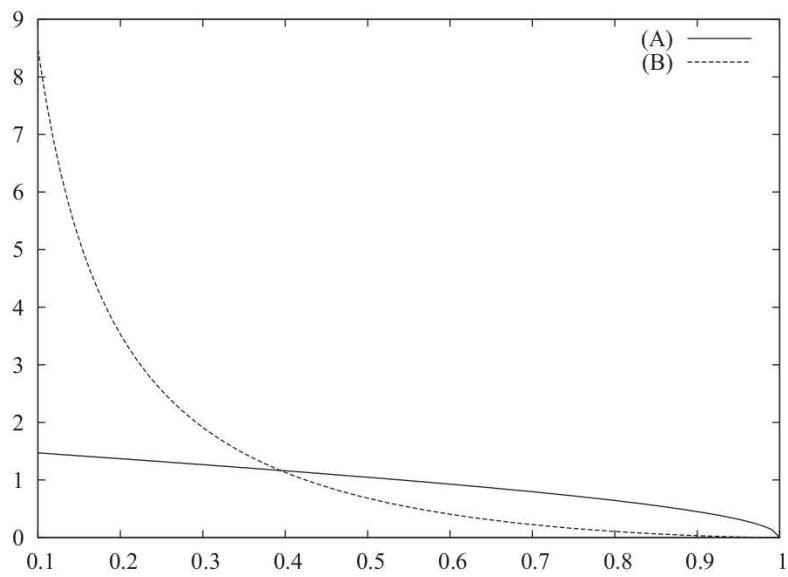

Figure 3. A comparison of the functionals $J_{1}(\omega) / 16 \pi$ denoted by (A) and $J_{2}(\omega) / 16 \pi$ by (B)

obtain the modulation equation

$$
\frac{\omega}{\mu} \frac{\partial J_{1}}{\partial \widetilde{t}}+\frac{k}{\mu} \frac{\partial J_{1}}{\partial \widetilde{x}}=-\frac{1}{2}\left\langle\frac{\partial u_{0}}{\partial \theta} F_{0}\right\rangle
$$

in which

$$
J_{1}(\omega(\widetilde{x}, \widetilde{t}))=\frac{\omega}{2}\left\langle\left(\frac{\partial u_{0}}{\partial \theta}\right)^{2}\right\rangle=16 \pi \arccos (\omega),
$$

provided that $J_{1}<8 \pi^{2}$.

\subsubsection{Solvability condition}

If we substitute the second solution $v_{2}$ into (3.11) and again exploit the parity of the terms, we have

in which

$$
\frac{\omega k}{\mu} \frac{\partial J_{2}}{\partial \widetilde{t}}+\frac{\omega^{2}}{\mu} \frac{\partial J_{2}}{\partial \widetilde{x}}=-\frac{1}{2}\left\langle\frac{\partial u_{0}}{\partial \phi} F_{0}\right\rangle
$$

$$
J_{2}(\omega(\widetilde{x}, \widetilde{t}))=\frac{1}{2 \omega}\left\langle\left(\frac{\partial u_{0}}{\partial \phi}\right)^{2}\right\rangle=16 \pi\left[\frac{\left(1-\omega^{2}\right)^{1 / 2}}{\omega}-\arccos (\omega)\right] .
$$

The functionals $J_{1}(\omega)$ and $J_{2}(\omega)$ are compared in Figure 3. It is noteworthy that although $J_{1}$ and $J_{2}$ are different quantities, they are directly related via $J_{2}=16 \pi \tan \left(J_{1} / 16 \pi\right)-J_{1}$.

\subsection{Necessary conditions}

On the long time scale and length scale, we have two unknowns $\omega$ and $k$. There are four equations for these two unknowns: two consistency conditions (3.2), an amplitude modulation equation (3.14) and a solvability condition (3.15). The two slowly varying parameters $\Psi$ and $\Lambda$ do not appear in these four equations: the equations for their modulation require consideration of the problem at $\mathcal{O}\left(\epsilon^{2}\right)$. For general perturbations, we would 


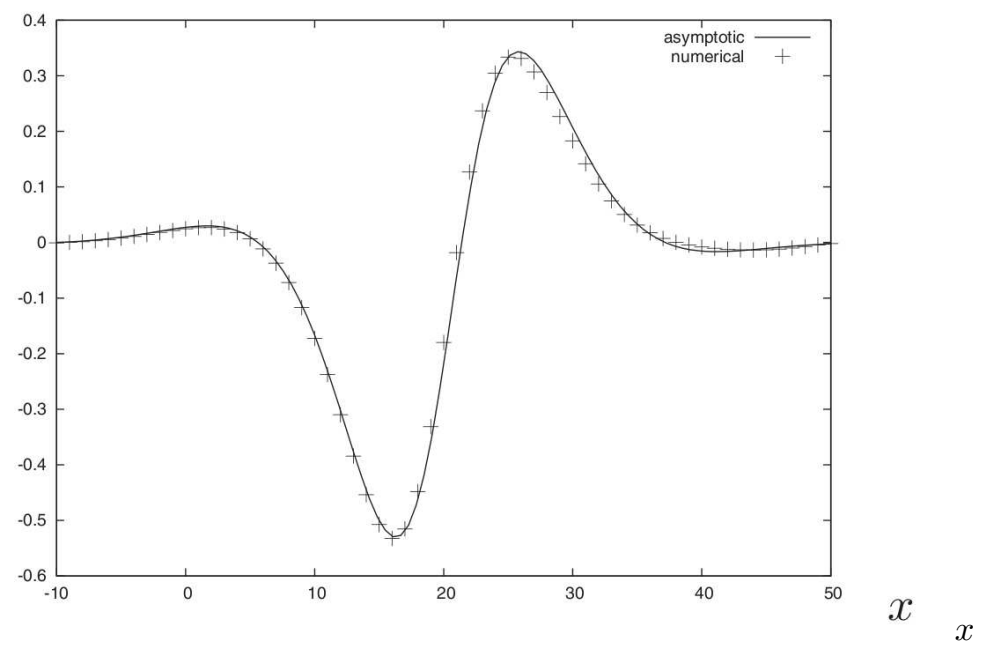

Figure 4. A comparison of the numerical solution of the discrete SG equation and the asymptotic solution using phase shifts independent of the long length scale.

not expect this overdetermined system to have a solution; however, we are interested in the special case in which $\omega$ and $k$ are constant. The system of four equations reduces to two necessary conditions for the approximation of breather solutions on discrete lattices

$$
\left\langle\frac{\partial u_{0}}{\partial \theta} F_{0}\right\rangle=0, \quad\left\langle\frac{\partial u_{0}}{\partial \phi} F_{0}\right\rangle=0
$$

In the continuum version (1.2), we have

$$
F_{0}=\frac{\omega^{4}}{\mu^{4}}\left[k^{4} \frac{\partial^{4} u_{0}}{\partial \theta^{4}}+4 k^{3} \frac{\partial^{4} u_{0}}{\partial \theta^{3} \partial \phi}+6 k^{2} \frac{\partial^{4} u_{0}}{\partial \theta^{2} \partial \phi^{2}}+4 k \frac{\partial^{4} u_{0}}{\partial \theta \partial \phi^{3}}+\frac{\partial^{4} u_{0}}{\partial \phi^{4}}\right] .
$$

The first, third and fifth terms on the right-hand side of (3.17) are even about $\theta+\Psi=n \pi$ and about $\phi+\Lambda=0$, whereas the second and fourth terms on the right-hand side of (3.17) are odd about $\theta+\Psi=n \pi$ and about $\phi+\Lambda=0$. Both necessary conditions (3.16) are met based on parity arguments. The necessary conditions for (1.3) and (1.4) are also met in a similar manner. We note that, in general, the slowly varying parameters $\Psi$ and $\Lambda$ will not be constant for these three non-dissipative perturbations.

The constancy of $k$ and $\omega$ is consistent with the inverse scattering analysis in [16]; however, the two approaches differ in that the phase shifts are predicted to be functions of the long length scale in this article. In order to investigate which of these approaches agrees with the numerical solutions, we consider the numerical solution of the discrete moving breather and the analytical solution of the continuum version with the initial conditions given by $\omega=\cos (\pi / 16), k=\sin (\pi / 16)$ and the phase shifts being zero. After one hundred time units, the numerical solution is compared with (3.4) except that the phase shifts are taken to be independent of the long length scale as in [16]. Figure 4 shows that the phase shifts are correct for $0 \leq x \leq 10$, but the phase shift is incorrect for $x \geq 20$. The phase shifts need to be slowly varying functions of space in order to obtain an accurate leading-order solution. 


\subsection{Damping}

The system of four equations ((3.2), (3.14) and (3.15)) for the two unknowns ( $\omega$ and $k$ ) on the long time scale and length scale provides little insight into the underlying physics. Accordingly, we again consider a perturbation which represents damping in the form $F=-\lambda u_{t}$ and $\lambda>0$. We have

$$
F_{0}=\frac{\lambda \omega^{2}}{\mu} \frac{\partial u_{0}}{\partial \theta}+\frac{\lambda k}{\mu} \frac{\partial u_{0}}{\partial \phi}
$$

which we substitute into (3.14) and (3.15) to obtain

$$
\omega \frac{\partial J_{1}}{\partial \widetilde{t}}+k \frac{\partial J_{1}}{\partial \widetilde{x}}=-\lambda \omega J_{1}
$$

and

$$
k \frac{\partial J_{2}}{\partial \widetilde{t}}+\omega \frac{\partial J_{2}}{\partial \widetilde{x}}=-\lambda k J_{2}
$$

respectively. After some algebraic manipulation, equations (3.2) may be rewritten as

$$
\frac{\partial k}{\partial \widetilde{t}}+\frac{\partial \omega}{\partial \widetilde{x}}=0, \quad k^{2} \frac{\partial \omega}{\partial \widetilde{t}}+2 k \omega \frac{\partial \omega}{\partial \widetilde{x}}-\omega^{2} \frac{\partial k}{\partial \widetilde{x}}=0 .
$$

The only solution is $k=0$. We have recovered the damped stationary breather. The damped moving breather should not be expected to exist in the presence of dissipation. Inverse scattering analysis also predicts substantially new effects with non-zero damping [16], but damped moving breathers are still predicted.

\section{Discussion}

Whilst the theory of Kuzmak-Luke has previously been applied to strongly nonlinear waves, these analyses have been restricted to the periodic case. Here, we have applied the theory to waves which are periodic in time, but solitary in space. To our knowledge this is the first application of this theory to such a system.

The sine-Gordon system is interesting, since it possesses both moving and stationary solutions, as well as both simple travelling waves and breather modes, which have an internal degree of freedom. For these more complex waves, we have found expressions for the wave action, which describe its dependence on frequency which, using multiple scales asymptotics, is permitted to evolve on a long time scale.

We have calculated expressions for special quantities of interest, namely the wave action, in the static and moving breather cases. In the static case there is just one quantity, $J$, which corresponds to the kinetic energy of the system. This is conserved in the case of the quasi-continuum sine-Gordon system, decreases exponentially for the damped sine-Gordon system and satisfies an inhomogeneous ordinary differential equation for the forced sine-Gordon system. In all cases, the solvability condition (2.17) is met, showing that static breather solutions give the correct leading-order behaviour on an order $1 / \epsilon$ time scale.

For the moving breather, there are two wave actions, corresponding to the kinetic energy $\left(J_{1}\right)$ and the elastic component of the potential energy $\left(J_{2}\right)$. In this case, we have two consistency conditions (3.2), an amplitude modulation equation (3.14) and a 
solvability condition (3.15). It is not possible to satisfy all of these in the case of a damped sine-Gordon system, so the decay of a moving breather in such a system is expected to be more complex than simply a moving breather with slowly varying amplitude, frequency and speed. A moving breather in a quasi-continuum sine-Gordon system, however, meets all the specified conditions on an order $1 / \epsilon$ time scale.

Recent experimental results on the long lifetimes of moving breathers in a layered crystal insulator will require more realistic models than the sine-Gordon system [27]. It is noteworthy that the technique applied in this article is quite general; it does not rely on the rare properties of integrability as in the case of the inverse scattering method. The method of Kuzmak-Luke may be readily extended to meet these new challenges. In particular, the methodology outlined in this paper is useful in the use of quasi-continuum methods, which rely on the approximation of a discrete system by a hierarchy of continuous ones using asymptotic techniques. The leading order continuum equation may have solutions with special properties, such as travelling waves or breathers. It is then desirable to know whether the original discrete system possesses solutions with similar properties. The above theory enables one to determine whether such solutions exist in the next order continuum asymptotic equation.

In summary, we have derived conditions under which the multiple scales asymptotic techniques used in previous works give consistent breather solutions. These conditions are given in Section 2.3 for the stationary breather and Section 3.3 for the moving breather and we have given illustrations of both cases when they are met and when they fail.

Acknowledgement. This project has benefited from the constructive comments of two anonymous referees.

\section{References}

[1] Birnir, B. 1994 Qualitative analysis of radiating breathers Comm. Pure Appl. Math. 47, 103-119.

[2] Birnir, B. 1994 Nonexistence of periodic solutions to hyperbolic partial differential equations In M. Gyllenberg and L. E. Persson (Eds.) Analysis, Algebra and Computers in Mathematical Research New York. Marcel Dekker.

[3] Boesch, R. \& Peyrard, M. 1991 Discreteness effects on a sine-Gordon breather Phys. Rev. $B$ 43, 8491-8508.

[4] Cisneros-Ake, L. A. 2013 Variational approximation for wave propagation in continuum and discrete media. Revista Mexicana de Fisica E, 59, 56-64.

[5] Collins, M. A. 1981 A quasi-continuum approximation for solitons in an atomic chain Chem. Phys. Lett. 77, 342-347.

[6] Collins, M. A. \& Rice, S. A. 1982 Some properties of large amplitude motion in an anharmonic chain with nearest neighbour interactions J. Chem. Phys. 77, 2607-2622.

[7] Dauxois, T. \& Peyrard, M. 1993 Energy localisation in nonlinear lattices Phys. Rev. Lett. 70, 3935-3938.

[8] Denzler, J. 1993 Nonpersistence of breather families for the perturbed sine Gordon equation Commun. Math. Phys. 158, 397-430.

[9] Dmitriev, S. V., Kevrekidis, P. G., Yoshikawa, N. 2006 Standard nearest-neighbour discretizations of Klein-Gordon models cannot preserve both energy and linear momentum, J. Phys. A, 39, 7217-7226.

[10] Drazin, P. G. \& Johnson, R. S. 1989 Solitons: an introduction. Cambridge. Cambridge University Press. 
[11] Englander, S. W., Kellenbach, N. R., Heeger, A. J., Krumhansl, J. A. \& Litwin, A. 1980 Nature of the open state in long polynucleotide double helices: possibility of soliton excitations Proc. Natl. Acad. Sci. 77, 7222-7226.

[12] Flach, S. \& Willis, C. 1993 Localized excitations in a discrete Klein-Gordon system Phys. Lett. A 181, 232-238.

[13] Friesecke, G. \& Wattis, J.A.D. 1994 Existence theorem for travelling waves on lattices, Commun. Math. Phys. 161, 391-418.

[14] Geicke, J. 1994 Logarithmic decay of $\phi^{4}$ breathers of energy $E \lesssim 1$ Phys. Rev. E 49, 35393542.

[15] Golubov, A., Serpuchenko, I. \& Ustinov, A. 1988 Dynamics of a Josephson fluxon in a long junction with inhomogeneities: theory and experiment Sov. Phys. JETP 67, 1256-1264.

[16] Karpman, V. I., Maslov, E. M. \& Solov'ev, V. V. 1983 Dynamics of bions in long Josephson junctions Sov. Phys. JETP 57, 167-173.

[17] Kevrekedis, P. G. 2003 On a class of discretizations of Hamiltonian nonlinear partial differential equations, Physica D 183, 68-86.

[18] Kevrekedis, P. G., Putkaradze, V., Rapti, Z. 2015 Non-holonomic constraints and their impact on discretizations of Klein-Gordon lattice dynamical models. arXiv.org: 1503.05516 [nlin.PS]

[19] Kuzmak, G. E. 1959 Asymptotic solutions of nonlinear second order differential equations with variable coefficients Prikl. Mat. Mekh. 23, 515-526 (Russian) J. Appl. Math. Mech. 23, 730-744.

[20] Luke, J. C. 1966 A perturbation method for nonlinear dispersive wave problems Proc. Roy. Soc. Lond. A 292, 403-412.

[21] Malomed, B. A. 1988 Dynamics of a fluxon in a long Josephson junction with a periodic lattice of inhomogeneities Phys. Rev. B 38, 9242-9244.

[22] Orfandis, S. J. 1979 Discrete solitons by the bilinear transformation Proc. IEEE 67, 175176.

[23] Peyrard, M. \& Bishop, A. R. 1989 Statistical mechanics of a nonlinear model for DNA denaturation Phys. Rev. Lett. 62, 2755-2758.

[24] Peyrard, M. \& Kruskal, M. 1984 Kink dynamics in the highly discrete sine-Gordon system Physica D 14, 88-102.

[25] Rosenau, P. 1986 Dynamics of nonlinear mass spring chains near the continuum limit Phys. Lett. A 118, 222-227.

[26] Rosenau, P. 1987 Dynamics of dense lattices Phys. Rev. A 36, 5868-5876.

[27] Russell, F. M. \& Eilbeck, J. C. 2007 Evidence for moving breathers in a layered crystal insulator at $300 \mathrm{~K}$ Europhys. Lett. 78, 10004.

[28] Salerno, M. 1991 Discrete models for DNA promoter dynamics Phys. Rev. A 44, 5292-5297.

[29] Segur, H. \& Kruskal, M. D. 1987 Nonexistence of small-amplitude breather solutions in $\phi^{4}$ theory Phys. Rev. Lett. 58, 747-750.

[30] Sмiтh, W. R. 2005 On the sensitivity of strongly nonlinear autonomous oscillators and oscillatory waves to small perturbations IMA J. Appl. Math. 70, 359-385.

[31] Sмith, W. R. 2010 Modulation equations for strongly nonlinear oscillations of an incompressible viscous drop J. Fluid Mech. 654, 141-159.

[32] WAttis, J. A. D. 1993 Approximations to solitary waves on lattices, II: quasi-continuum approximations for fast and slow waves J. Phys. A; Math. Gen. 26, 1193-1209.

[33] Wattis, J. A. D. 1995 Variational approximations to breather modes in the discrete sineGordon equation Physica D 82, 333-339.

[34] Wattis, J. A. D. 1996 Variational approximations to breathers in the discrete sine-Gordon equation II: moving breathers and Peierls-Nabarro energies Nonlinearity 9, 1583-1598.

[35] Wattis, J. A. D. 1998 Stationary breather modes of generalised nonlinear Klein-Gordon lattices J. Phys. A; Math. Gen. 31, 3301-3323.

[36] Whitham, G. B. 1974 Linear and Nonlinear Waves. New York. John Wiley \& Sons.

[37] Yakushevich, L. V. 1998 Nonlinear Physics of DNA. Chichester. John Wiley \& Sons. 
Dmitriev et al. [9]

Kevrekedis et al. [18] 